\title{
Disjunctive Cuts for Cross-Sections of the Second-Order Cone
}

\author{
Sercan Ylldız * Gérard Cornuéjols ${ }^{\dagger}$
}

June 10, 2014, revised May 25, 2015

\begin{abstract}
In this paper we study general two-term disjunctions on affine cross-sections of the secondorder cone. Under some mild assumptions, we derive a closed-form expression for a convex inequality that is valid for such a disjunctive set, and we show that this inequality is sufficient to characterize the closed convex hull of all two-term disjunctions on ellipsoids and paraboloids and a wide class of two-term disjunctions - including split disjunctions - on hyperboloids. Our approach relies on the work of Kılınç-Karzan and Yıldız which considers general two-term disjunctions on the second-order cone.
\end{abstract}

Keywords: Mixed-integer conic programming, second-order cone programming, cutting planes, disjunctive cuts

\section{Introduction}

In this paper we consider the mixed-integer second-order conic set

$$
S:=\left\{x \in \mathbb{L}^{n}: A x=b, x_{j} \in \mathbb{Z} \forall j \in J\right\}
$$

where $\mathbb{L}^{n}$ is the $n$-dimensional second-order cone $\mathbb{L}^{n}:=\left\{x \in \mathbb{R}^{n}:\left\|\left(x_{1} ; \ldots ; x_{n-1}\right)\right\| \leq x_{n}\right\}, A$ is an $m \times n$ real matrix of full row rank, $d$ and $b$ are real vectors of appropriate dimensions, $J \subseteq\{1, \ldots, n\}$, and $\|\cdot\|$ denotes the Euclidean norm. The set $S$ appears as the feasible solution set or a relaxation thereof in mixed-integer second order cone programming problems. Because the structure of $S$ can be very complicated, a first approach to solving

$$
\sup \left\{d^{\top} x: x \in S\right\} .
$$

entails solving the relaxed problem obtained after dropping the integrality requirements on the variables:

$$
\sup \left\{d^{\top} x: x \in C\right\} \text { where } C:=\left\{x \in \mathbb{L}^{n}: A x=b\right\} .
$$

The set $C$ is called the natural continuous relaxation of $S$. Unfortunately, the continuous relaxation $C$ is often a poor approximation to the mixed-integer conic set $S$, and tighter formulations are needed for the development of practical strategies for solving (1). An effective way to improve the approximation quality of the continuous relaxation $C$ is to strengthen it with additional convex inequalities that are valid for $S$ but not for the whole of $C$. Such valid inequalities can be

\footnotetext{
${ }^{*}$ Tepper School of Business, Carnegie Mellon University, Pittsburgh, PA, syildiz@andrew.cmu.edu.

${ }^{\dagger}$ Tepper School of Business, Carnegie Mellon University, Pittsburgh, PA, gc0v@andrew.cmu.edu.
} 
derived by exploiting the integrality of the variables $x_{j}, j \in J$, and enhancing $C$ with linear two-term disjunctions $l_{1}^{\top} x \geq l_{1,0} \vee l_{2}^{\top} x \geq l_{2,0}$ that are satisfied by all solutions in $S$. Valid inequalities that are obtained from disjunctions using this approach are known as disjunctive cuts. In this paper we study two-term disjunctions on the set $C$ and give closed-form expressions for the strongest disjunctive cuts that can be obtained from such disjunctions.

Disjunctive cuts were introduced by Balas in the context of mixed-integer linear programming [3] and have since been the cornerstone of theoretical and practical achievements in integer programming. There has been a lot of recent interest in extending disjunctive cutting-plane theory from the domain of mixed-integer linear programming to that of mixed-integer conic programming [2, 7, 9, 11, 12, 18. Kılınç-Karzan [13] studied minimal valid linear inequalities for general disjunctive conic sets and showed that these are sufficient to describe the associated closed convex hull under a mild technical assumption. Bienstock and Michalka [6] studied the characterization and separation of linear inequalities that are valid for the epigraph of a convex, differentiable function whose domain is restricted to the complement of a convex set. On the other hand, several papers in the last few years have focused on deriving closed-form expressions for nonlinear convex inequalities that fully describe the convex hull of a disjunctive second-order conic set in the space of the original variables. Dadush et al. [10 and Andersen and Jensen [1] derived split cuts for ellipsoids and the second-order cone, respectively. Modaresi et al. extended these results to split disjunctions on cross-sections of the second-order cone [16] and compared the effectiveness of split cuts against conic MIR inequalities and extended formulations [15]. For disjoint two-term disjunctions on cross-sections of the second-order cone and under the assumption that $\left\{x \in C: l_{1}^{\top} x=l_{1,0}\right\}$ and $\left\{x \in C: l_{2}^{\top} x=l_{2,0}\right\}$ are bounded, Belotti et al. [4, 5] proved that there exists a unique cone which describes the convex hull of the disjunction. They also identified a procedure for identifying this cone when $C$ is an ellipsoid. Using the structure of minimal valid linear inequalities, Kılınç-Karzan and Yıldız [14] derived a family of convex inequalities which describes the convex hull of a general two-term disjunction on the whole second-order cone. In this paper, we pursue a similar goal: We study general two-term disjunctions on a cross-section $C$ of the second-order cone, namely $C=\left\{x \in \mathbb{L}^{n}: A x=b\right\}$. Given a disjunction $l_{1}^{\top} x \geq l_{1,0} \vee l_{2}^{\top} x \geq l_{2,0}$ on $C$, we let

$$
C_{1}:=\left\{x \in C: l_{1}^{\top} x \geq l_{1,0}\right\} \quad \text { and } \quad C_{2}:=\left\{x \in C: l_{2}^{\top} x \geq l_{2,0}\right\} .
$$

In order to derive the tightest disjunctive cuts that can be obtained for $S$ from the disjunction $C_{1} \cup C_{2}$, we study the closed convex hull $\overline{\operatorname{conv}}\left(C_{1} \cup C_{2}\right)$. In particular, we are interested in convex inequalities that may be added to the description of $C$ to obtain a characterization of $\overline{\operatorname{conv}}\left(C_{1} \cup C_{2}\right)$. Our starting point is the paper [14] about two-term disjunctions on the second-order cone $\mathbb{L}^{n}$. We extend the main result of [14] to cross-sections of the second-order cone. Such cross-sections include ellipsoids, paraboloids, and hyperboloids as special cases. Our results generalize the work of [10, 16] on split disjunctions on cross-sections of the second-order cone and [4] on disjoint two-term disjunctions on ellipsoids. We note here that general results on convexifying the intersection of a cross-section of the second-order cone with a non-convex cone defined by a single homogeneous quadratic were recently obtained independently in [8].

We first show in Section 2 that the continuous relaxation $C$ can be assumed to be the intersection of a lower-dimensional second-order cone with a single hyperplane. In Section 3 , we give a complete description of the convex hull of a homogeneous two-term disjunction on the whole second-order cone. In Section 4, we prove our main result, Theorem 3 , characterizing $\overline{\operatorname{conv}}\left(C_{1} \cup C_{2}\right)$ under certain conditions. We end the paper with two examples which illustrate the applicability of Theorem 3 . 
In this section, we show that the continuous relaxation $C$ can be assumed to be the intersection of a lower-dimensional second-order cone with a single hyperplane. Let $E:=\left\{x \in \mathbb{R}^{n}: A x=b\right\}$ so that $C=\mathbb{L}^{n} \cap E$. We are going to use the following lemma to simplify our analysis.

Lemma 1. Let $V$ be a p-dimensional linear subspace of $\mathbb{R}^{n}$. The intersection $\mathbb{L}^{n} \cap V$ is either the origin, a half-line, or a bijective linear transformation of $\mathbb{L}^{p}$.

See Section 2.1 of [5] for a similar result. We do not give a formal proof of Lemma 1 but just note that it can be obtained by observing that the second-order cone is the conic hull of a (one dimension smaller) sphere, and that the intersection of a sphere with an affine space is either empty, a single point (when the affine space intersects the sphere but not its interior), or a lower dimensional sphere of the same dimension as the affine space (when the affine space intersects the interior of the sphere).

Lemma 1 implies that, when $b=0, C$ is either the origin, a half-line, or a bijective linear transformation of $\mathbb{L}^{n-m}$. The closed convex hull $\overline{\operatorname{conv}}\left(C_{1} \cup C_{2}\right)$ can be described easily when $C$ is a single point or a half-line. Furthermore, the problem of characterizing $\overline{\operatorname{conv}}\left(C_{1} \cup C_{2}\right)$ when $C$ is a bijective linear transformation of $\mathbb{L}^{n-m}$ can be reduced to that of convexifying an associated two-term disjunction on $\mathbb{L}^{n-m}$. We refer the reader to [14 for a detailed study of the closed convex hulls of two-term disjunctions on the second-order cone.

In the remainder, we focus on the case $b \neq 0$. Note that, whenever this is the case, we can permute and normalize the rows of $(A, b)$ so that its last row reads $\left(a_{m}^{\top}, 1\right)$, and subtracting a multiple of $\left(a_{m}^{\top}, 1\right)$ from the other rows if necessary, we can write the remaining rows of $(A, b)$ as $(\tilde{A}, 0)$. Therefore, we can assume without any loss of generality that all components of $b$ are zero except the last one. Isolating the last row of $(A, b)$ from the others, we can then write

$$
E=\left\{x \in \mathbb{R}^{n}: \tilde{A} x=0, a_{m}^{\top} x=1\right\}
$$

Let $V:=\left\{x \in \mathbb{R}^{n}: \tilde{A} x=0\right\}$. By Lemma 1, $\mathbb{L}^{n} \cap V$ is the origin, a half-line, or a bijective linear transformation of $\mathbb{L}^{n-m+1}$. Again, the first two cases are easy and not of interest in our analysis. In the last case, we can find a matrix $D$ whose columns form an orthonormal basis for $V$ and define a nonsingular matrix $H$ such that $\left\{y \in \mathbb{R}^{n-m+1}: D y \in \mathbb{L}^{n}\right\}=H \mathbb{L}^{n-m+1}$. Then 
we can represent $C$ equivalently as

$$
\begin{aligned}
C & =\left\{x \in \mathbb{L}^{n}: x=D y, a_{m}^{\top} x=1\right\} \\
& =D\left\{y \in \mathbb{R}^{n-m+1}: D y \in \mathbb{L}^{n}, a_{m}^{\top} D y=1\right\} \\
& =D\left\{y \in \mathbb{R}^{n-m+1}: y \in H \mathbb{L}^{n-m+1}, a_{m}^{\top} D y=1\right\} \\
& =D H\left\{z \in \mathbb{L}^{n-m+1}: a_{m}^{\top} D H z=1\right\} .
\end{aligned}
$$

The set $C=\mathbb{L}^{n} \cap E$ is a bijective linear transformation of $\left\{z \in \mathbb{L}^{n-m+1}: a_{m}^{\top} D H z=1\right\}$. Furthermore, the same linear transformation maps any two-term disjunction in $\left\{z \in \mathbb{L}^{n-m+1}\right.$ : $\left.a_{m}^{\top} D H z=1\right\}$ to a two-term disjunction in $C$ and vice versa. Thus, without any loss of generality, we can take $m=1$ in (1) and study the problem of describing $\overline{\operatorname{conv}}\left(C_{1} \cup C_{2}\right)$ where

$$
\begin{gathered}
C=\left\{x \in \mathbb{L}^{n}: a^{\top} x=1\right\}, \\
C_{1}=\left\{x \in C: l_{1}^{\top} x \geq l_{1,0}\right\}, \quad \text { and } \quad C_{2}=\left\{x \in C: l_{2}^{\top} x \geq l_{2,0}\right\} .
\end{gathered}
$$

In Section 4 we will give a full description of $\overline{\operatorname{conv}}\left(C_{1} \cup C_{2}\right)$ under certain conditions.

\section{Homogeneous Two-Term Disjunctions on the Second-Order Cone}

In this section, we study the convex hull of a homogeneous two-term disjunction $c_{1}^{\top} x \geq 0 \vee c_{2}^{\top} x \geq$ 0 on the second-order cone. Let

$$
Q_{1}:=\left\{x \in \mathbb{L}^{n}: c_{1}^{\top} x \geq 0\right\} \quad \text { and } \quad Q_{2}:=\left\{x \in \mathbb{L}^{n}: c_{2}^{\top} x \geq 0\right\} .
$$

The main result of this section characterizes $\operatorname{conv}\left(Q_{1} \cup Q_{2}\right)$. Note that $Q_{1}$ and $Q_{2}$ are closed, convex, pointed cones; therefore, $\operatorname{conv}\left(Q_{1} \cup Q_{2}\right)$ is always closed (see, e.g., Rockafellar [17, Corollary 9.1.3]).

When $Q_{1} \subseteq Q_{2}$, we have conv $\left(Q_{1} \cup Q_{2}\right)=Q_{2}$. Similarly, when $Q_{1} \supseteq Q_{2}$, we have $\operatorname{conv}\left(Q_{1} \cup\right.$ $\left.Q_{2}\right)=Q_{1}$. In the remainder of this section, we focus on the case where $Q_{1} \nsubseteq Q_{2}$ and $Q_{1} \nsupseteq Q_{2}$.

Assumption 1. $Q_{1} \nsubseteq Q_{2}$ and $Q_{1} \nsupseteq Q_{2}$.

We also make the following technical assumption.

Assumption 2. $Q_{1} \cap \operatorname{int} \mathbb{L}^{n} \neq \emptyset$ and $Q_{2} \cap \operatorname{int} \mathbb{L}^{n} \neq \emptyset$.

This assumption will be useful later when we use Theorem 1 whose proof relies on conic duality.

By Assumption 1, we have $Q_{1}, Q_{2} \subsetneq \mathbb{L}^{n}$, and by Assumption 2, we have that $Q_{1}$ and $Q_{2}$ are full-dimensional. This implies $c_{1}, c_{2} \notin \pm \mathbb{L}^{n}$, or equivalently $\left\|\tilde{c}_{i}\right\|^{2}>c_{i, n}^{2}$, for $i \in\{1,2\}$. By scaling $c_{1}$ and $c_{2}$ with appropriate positive scalars if necessary, we may assume without any loss of generality that

$$
\left\|\tilde{c}_{1}\right\|^{2}-c_{1, n}^{2}=\left\|\tilde{c}_{2}\right\|^{2}-c_{2, n}^{2}=1 .
$$

These have the following consequences. 


$$
\begin{aligned}
\mathcal{M} & :=\left\|\tilde{c}_{1}\right\|^{2}-c_{1, n}^{2}-\left(\left\|\tilde{c}_{2}\right\|^{2}-c_{2, n}^{2}\right)=0 \\
\mathcal{N} & :=\left\|\tilde{c}_{1}-\tilde{c}_{2}\right\|^{2}-\left(c_{1, n}-c_{2, n}\right)^{2}=2-2\left(\tilde{c}_{1}^{\top} \tilde{c}_{2}-c_{1, n} c_{2, n}\right) .
\end{aligned}
$$

Remark 2. Let $Q_{1}$ and $Q_{2}$, defined as in (3), satisfy Assumption 1 . Then we have $c_{1}-c_{2} \notin \pm \mathbb{L}^{n}$. Indeed, $c_{1}-c_{2} \in \mathbb{L}^{n}$ implies that $\left(c_{1}-c_{2}\right)^{\top} x \geq 0$ for all $x \in \mathbb{L}^{n}$, and this implies $C_{1} \subseteq C_{2}$; similarly, $c_{2}-c_{1} \in \mathbb{L}^{n}$ implies $C_{2} \subseteq C_{1}$. Hence,

$$
\mathcal{N}=\left\|\tilde{c}_{1}-\tilde{c}_{2}\right\|^{2}-\left(c_{1, n}-c_{2, n}\right)^{2}>0
$$

The following result from [14] gives a valid convex inequality for $\operatorname{conv}\left(Q_{1} \cup Q_{2}\right)$.

Theorem 1 ([14], Theorem 3 and Remark 2). Let $Q_{1}$ and $Q_{2}$ be defined as in (3). Suppose Assumptions 1 and 2 hold. Then the inequality

$$
-\left(c_{1}+c_{2}\right)^{\top} x \leq \sqrt{\left(\left(c_{1}-c_{2}\right)^{\top} x\right)^{2}+\mathcal{N}\left(x_{n}^{2}-\|\tilde{x}\|^{2}\right)}
$$

is valid for $\operatorname{conv}\left(Q_{1} \cup Q_{2}\right)$. Furthermore, this inequality is convex in $\mathbb{L}^{n}$.

The next proposition shows that (5) can be written in conic quadratic form in $\mathbb{L}^{n}$ except in the region where both clauses of the disjunction are satisfied. Its proof is a simple extension of the proofs of Propositions 3 and 4 in [14] and therefore omitted. Let

$$
r:=\left(\begin{array}{c}
\tilde{c}_{1}-\tilde{c}_{2} \\
-c_{1, n}+c_{2, n}
\end{array}\right) .
$$

Proposition 1 ([14, Propositions 3 and 4). Let $Q_{1}$ and $Q_{2}$ be defined as in (3). Suppose Assumptions 1 and 2 hold. Let $x^{\prime} \in \mathbb{L}^{n}$ be such that $c_{1}^{\top} x^{\prime} \leq 0$ or $c_{2}^{\top} x^{\prime} \leq 0$. Then the following statements are equivalent:

i) $x^{\prime}$ satisfies (5).

ii) $x^{\prime}$ satisfies the conic quadratic inequality

$$
\mathcal{N} x-2\left(c_{1}^{\top} x\right) r \in \mathbb{L}^{n}
$$

iii) $x^{\prime}$ satisfies the conic quadratic inequality

$$
\mathcal{N} x+2\left(c_{2}^{\top} x\right) r \in \mathbb{L}^{n} .
$$

Remark 3. When $c_{1}$ and $c_{2}$ satisfy (4), the inequalities (6) and (7) describe a cylindrical secondorder cone whose lineality space contains $\operatorname{span}\{r\}$. This follows from Remark 1 by observing that

$$
\mathcal{N}=2-2\left(\tilde{c}_{1}^{\top} \tilde{c}_{2}-c_{1, n} c_{2, n}\right)=2 c_{1}^{\top} r=-2 c_{2}^{\top} r .
$$

The next theorem is the main result of this section. It shows that the inequality (5) is in fact sufficient to describe $\operatorname{conv}\left(Q_{1} \cup Q_{2}\right)$ when $c_{1}$ and $c_{2}$ are scaled so that they satisfy (4). Because this assumption is without any loss of generality, our result settles one of the cases left open by Kılınç-Karzan and Yıldız [14, where the right-hand-sides of both terms of the disjunction are zero in 3 . 
Theorem 2. Let $Q_{1}$ and $Q_{2}$ be defined as in (3). Suppose Assumptions 1 and 2 hold. Assume that $c_{1}$ and $c_{2}$ have been scaled so that they satisfy (4). Then

$$
\operatorname{conv}\left(Q_{1} \cup Q_{2}\right)=\left\{x \in \mathbb{L}^{n}: x \text { satisfies }(5)\right\} \text {. }
$$

Proof. Let $D$ denote the set on the right-hand side of (8). We already know that (5) is valid for $\operatorname{conv}\left(Q_{1} \cup Q_{2}\right)$. Hence, $\operatorname{conv}\left(Q_{1} \cup Q_{2}\right) \subseteq D$. Let $x^{\prime} \in D$. If $x^{\prime} \in Q_{1} \cup Q_{2}$, then clearly $x^{\prime} \in \operatorname{conv}\left(Q_{1} \cup Q_{2}\right)$. Therefore, suppose $x^{\prime} \in \mathbb{L}^{n} \backslash\left(Q_{1} \cup Q_{2}\right)$ is a point that satisfies (5). By Proposition 1, $x^{\prime}$ satisfies

$$
\mathcal{N} x^{\prime}-2\left(c_{1}^{\top} x^{\prime}\right) r \in \mathbb{L}^{n} \text { and } \mathcal{N} x^{\prime}+2\left(c_{2}^{\top} x^{\prime}\right) r \in \mathbb{L}^{n} .
$$

We are going to show that $x^{\prime}$ belongs to $\operatorname{conv}\left(Q_{1} \cup Q_{2}\right)$.

By Remarks 2 and $3,0<\mathcal{N}=2 c_{1}^{\top} r=-2 c_{2}^{\top} r$. Let

$$
\begin{aligned}
& \alpha_{1}:=\frac{-c_{1}^{\top} x^{\prime}}{c_{1}^{\top} r}, \quad \alpha_{2}:=\frac{-c_{2}^{\top} x^{\prime}}{c_{2}^{\top} r}, \\
& x_{1}:=x^{\prime}+\alpha_{1} r, \quad x_{2}:=x^{\prime}+\alpha_{2} r .
\end{aligned}
$$

It is not difficult to see that $c_{1}^{\top} x_{1}=c_{2}^{\top} x_{2}=0$. Furthermore, $x^{\prime} \in \operatorname{conv}\left\{x_{1}, x_{2}\right\}$ because $\alpha_{2}<0<\alpha_{1}$. Therefore, the only thing we need to show is $x_{1}, x_{2} \in \mathbb{L}^{n}$. By Remark 3

$$
\mathcal{N} r-2\left(c_{1}^{\top} r\right) r=\mathcal{N} r+2\left(c_{2}^{\top} r\right) r=0 .
$$

Hence,

$$
\begin{aligned}
& \mathcal{N} x_{1}-2\left(c_{1}^{\top} x_{1}\right) r=\mathcal{N} x^{\prime}-2\left(c_{1}^{\top} x^{\prime}\right) r \in \mathbb{L}^{n} \quad \text { and } \\
& \mathcal{N} x_{2}+2\left(c_{2}^{\top} x_{2}\right) r=\mathcal{N} x^{\prime}+2\left(c_{2}^{\top} x^{\prime}\right) r \in \mathbb{L}^{n} .
\end{aligned}
$$

Now observing that $c_{1}^{\top} x_{1}=c_{2}^{\top} x_{2}=0$ and $\mathcal{N}>0$ shows $x_{1}, x_{2} \in \mathbb{L}^{n}$. This proves $x_{1} \in Q_{1}$ and $x_{2} \in Q_{2}$.

In the next section, we will show that the inequality (5) can also be used to characterize $\overline{\operatorname{conv}}\left(C_{1} \cup C_{2}\right)$ where $C_{1}$ and $C_{2}$ are defined as in (2).

\section{Two-Term Disjunctions on Cross-Sections of the Second- Order Cone}

\subsection{The Main Result}

Consider $C, C_{1}$, and $C_{2}$ defined as in (2). The set $C$ is an ellipsoid when $a \in \operatorname{int} \mathbb{L}^{n}$, a paraboloid when $a \in \operatorname{bd} \mathbb{L}^{n}$, a hyperboloid when $a \notin \pm \mathbb{L}^{n}$, and empty when $a \in-\mathbb{L}^{n}$. In this section, we prove our main result, Theorem 3 , which characterizes $\overline{\operatorname{conv}}\left(C_{1} \cup C_{2}\right)$ under some mild conditions.

When $C_{1} \subseteq C_{2}$, we have $\overline{\operatorname{conv}}\left(C_{1} \cup C_{2}\right)=C_{2}$. Similarly, when $C_{1} \supseteq C_{2}$, we have $\overline{\operatorname{conv}}\left(C_{1} \cup\right.$ $\left.C_{2}\right)=C_{1}$. In the remainder we concentrate on the case where $C_{1} \nsubseteq C_{2}$ and $C_{1} \nsupseteq C_{2}$.

Assumption 3. $C_{1} \nsubseteq C_{2}$ and $C_{1} \nsupseteq C_{2}$.

We also make the following assumption. 
This assumption will be useful later when we again use Theorem 1 whose proof relies on conic duality. The following simple observation underlies our approach.

Observation 1. Let $C, C_{1}$, and $C_{2}$ be defined as in (2). Then $C_{1}=\left\{x \in C:\left(\beta_{1} l_{1}+\gamma_{1} a\right)^{\top} x \geq\right.$ $\left.\beta_{1} l_{1,0}+\gamma_{1}\right\}$ for any $\beta_{1}>0$ and $\gamma_{1} \in \mathbb{R}$. Similarly, $C_{2}=\left\{x \in C:\left(\beta_{2} l_{2}+\gamma_{2} a\right)^{\top} x \geq \beta_{2} l_{2,0}+\gamma_{2}\right\}$ for any $\beta_{2}>0$ and $\gamma_{2} \in \mathbb{R}$.

Observation 1 allows us to conclude

$$
C_{1}=\left\{x \in C:\left(l_{1}-l_{1,0} a\right)^{\top} x \geq 0\right\} \quad \text { and } \quad C_{2}=\left\{x \in C:\left(l_{2}-l_{2,0} a\right)^{\top} x \geq 0\right\} .
$$

By Assumption 3, we have $C_{1}, C_{2} \subsetneq C$, and by Assumption 4, we have $\operatorname{dim} C_{1}=\operatorname{dim} C_{2}=n-1$. This implies $l_{i}-l_{i, 0} a \notin \pm \mathbb{L}^{n}$, or equivalently $\left\|\tilde{l}_{i}-l_{i, 0} \tilde{a}\right\|^{2}>\left(l_{i, n}-l_{i, 0} a_{n}\right)^{2}$, for $i \in\{1,2\}$. Let

$$
c_{i}:=\lambda_{i}\left(l_{i}-l_{i, 0} a\right) \text { where } \lambda_{i}:=\frac{1}{\sqrt{\left\|\tilde{l}_{i}-l_{i, 0} \tilde{a}\right\|^{2}-\left(l_{i, n}-l_{i, 0} a_{n}\right)^{2}}} \text { for } i \in\{1,2\} .
$$

Because $\lambda_{1}, \lambda_{2}>0$, we can write

$$
C_{1}=\left\{x \in C: c_{1}^{\top} x \geq 0\right\} \quad \text { and } \quad C_{2}=\left\{x \in C: c_{2}^{\top} x \geq 0\right\} .
$$

This scaling ensures that $c_{1}$ and $c_{2}$ satisfy (4).

Let $Q_{1}$ and $Q_{2}$ be the relaxations of $C_{1}$ and $C_{2}$ to the whole cone $\mathbb{L}^{n}$ :

$$
Q_{1}:=\left\{x \in \mathbb{L}^{n}: c_{1}^{\top} x \geq 0\right\} \quad \text { and } \quad Q_{2}:=\left\{x \in \mathbb{L}^{n}: c_{2}^{\top} x \geq 0\right\} .
$$

It is clear that $Q_{1}$ and $Q_{2}$ satisfy Assumptions $1+2$ because $C_{1}$ and $C_{2}$ satisfy Assumptions 3 4. Define $\mathcal{N}, \mathcal{M}$, and $r$ as in Section 3 using $c_{1}$ and $c_{2}$. Noting that $Q_{1}$ and $Q_{2}$ satisfy Assumptions 1, 2 and $c_{1}$ and $c_{2}$ satisfy (4), all results of Section 3 hold for $Q_{1}$ and $Q_{2}$. In particular, Theorem 1 implies that the inequality (5) is valid for $\overline{\operatorname{conv}}\left(C_{1} \cup C_{2}\right)$. In Theorem 3 , we are going to show that (5) is also sufficient to describe $\overline{\operatorname{conv}}\left(C_{1} \cup C_{2}\right)$ when the sets $C_{1}$ and $C_{2}$ satisfy certain conditions. The proof of Theorem 3 requires the following technical lemma.

Lemma 2. Let $C_{1}$ and $C_{2}$ be defined as in (2). Suppose Assumptions 3 and 4 hold. Let $c_{1}$ and $c_{2}$ be defined as in (10). Suppose $a^{\top} r \neq 0$, and let $x^{*}:=\frac{r}{a^{\top} r}$. Let $x^{\prime} \in C \backslash\left(C_{1} \cup C_{2}\right)$ satisfy (5).

a) If $a^{\top} r>0$, then $c_{1}^{\top}\left(x^{\prime}-x^{*}\right)<0$. If in addition

$$
\begin{gathered}
\left(a+\text { cone }\left\{c_{1}, c_{2}\right\}\right) \cap \mathbb{L}^{n} \neq \emptyset, \quad \text { or } \quad\left(-a+\operatorname{cone}\left\{c_{1}, c_{2}\right\}\right) \cap \mathbb{L}^{n} \neq \emptyset, \quad \text { or } \\
\left(-a+\operatorname{cone}\left\{c_{2}\right\}\right) \cap-\mathbb{L}^{n} \neq \emptyset,
\end{gathered}
$$

then $c_{2}^{\top}\left(x^{\prime}-x^{*}\right) \geq 0$.

b) If $a^{\top} r<0$, then $c_{2}^{\top}\left(x^{\prime}-x^{*}\right)<0$. If in addition

$$
\begin{gathered}
\left(a+\text { cone }\left\{c_{1}, c_{2}\right\}\right) \cap \mathbb{L}^{n} \neq \emptyset, \quad \text { or } \quad\left(-a+\operatorname{cone}\left\{c_{1}, c_{2}\right\}\right) \cap \mathbb{L}^{n} \neq \emptyset, \quad \text { or } \\
\left(-a+\operatorname{cone}\left\{c_{1}\right\}\right) \cap-\mathbb{L}^{n} \neq \emptyset,
\end{gathered}
$$

then $c_{1}^{\top}\left(x^{\prime}-x^{*}\right) \geq 0$. 
Proof. By Remarks 2 and $3, \mathcal{N}=2 c_{1}^{\top} r=-2 c_{2}^{\top} r>0$. From this, we get

$$
\begin{aligned}
\mathcal{N} x^{*}-2\left(c_{1}^{\top} x^{*}\right) r & =\frac{1}{a^{\top} r}\left(\mathcal{N}-2 c_{1}^{\top} r\right) r=0, \\
\mathcal{N} x^{*}+2\left(c_{2}^{\top} x^{*}\right) r & =\frac{1}{a^{\top} r}\left(\mathcal{N}+2 c_{2}^{\top} r\right) r=0 .
\end{aligned}
$$

Furthermore, $a^{\top} x^{\prime}=a^{\top} x^{*}=1$.

a) Having $x^{\prime} \notin C_{1}$ implies $c_{1}^{\top} x^{\prime}<0$. Furthermore, it follows from $c_{1}^{\top} r=\frac{\mathcal{N}}{2}>0$ that

$$
c_{1}^{\top} x^{*}=\frac{c_{1}^{\top} r}{a^{\top} r}>0
$$

Thus, we get $c_{1}^{\top}\left(x^{\prime}-x^{*}\right)<0$.

Now suppose $\left(a+\operatorname{cone}\left\{c_{1}, c_{2}\right\}\right) \cap \mathbb{L}^{n} \neq \emptyset$. Then there exist $\lambda \geq 0$ and $0 \leq \theta \leq 1$ such that $a+\lambda\left(\theta c_{1}+(1-\theta) c_{2}\right) \in \mathbb{L}^{n}$. The point $x^{\prime}$ does not belong to either $C_{1}$ or $C_{2}$ and satisfies (5). By Proposition 1, it satisfies (7) as well. Using (14), we can write

$$
\mathcal{N}\left(x^{\prime}-x^{*}\right)+2 c_{2}^{\top}\left(x^{\prime}-x^{*}\right) r \in \mathbb{L}^{n} \text {. }
$$

Because $\mathbb{L}^{n}$ is self-dual, we get

$$
\begin{aligned}
0 & \leq\left(a+\lambda\left(\theta c_{1}+(1-\theta) c_{2}\right)\right)^{\top}\left(\mathcal{N}\left(x^{\prime}-x^{*}\right)+2 c_{2}^{\top}\left(x^{\prime}-x^{*}\right) r\right) \\
& =2 c_{2}^{\top}\left(x^{\prime}-x^{*}\right) a^{\top} r+\lambda\left(\theta c_{1}+(1-\theta) c_{2}\right)^{\top}\left(\mathcal{N}\left(x^{\prime}-x^{*}\right)+2 c_{2}^{\top}\left(x^{\prime}-x^{*}\right) r\right) \\
& =2 c_{2}^{\top}\left(x^{\prime}-x^{*}\right) a^{\top} r+\lambda \theta\left(c_{1}-c_{2}\right)^{\top}\left(\mathcal{N}\left(x^{\prime}-x^{*}\right)+2 c_{2}^{\top}\left(x^{\prime}-x^{*}\right) r\right)+\lambda c_{2}^{\top}\left(x^{\prime}-x^{*}\right)\left(\mathcal{N}+2 c_{2}^{\top} r\right) \\
& =2 c_{2}^{\top}\left(x^{\prime}-x^{*}\right) a^{\top} r+\lambda \theta\left(c_{1}-c_{2}\right)^{\top}\left(\mathcal{N}\left(x^{\prime}-x^{*}\right)+2 c_{2}^{\top}\left(x^{\prime}-x^{*}\right) r\right) \\
& =2 c_{2}^{\top}\left(x^{\prime}-x^{*}\right) a^{\top} r+\lambda \theta\left(\mathcal{N}\left(c_{1}-c_{2}\right)^{\top}\left(x^{\prime}-x^{*}\right)+2 c_{2}^{\top}\left(x^{\prime}-x^{*}\right)\left(c_{1}-c_{2}\right)^{\top} r\right) \\
& =2 c_{2}^{\top}\left(x^{\prime}-x^{*}\right) a^{\top} r+\lambda \theta\left(\mathcal{N}\left(c_{1}+c_{2}\right)^{\top}\left(x^{\prime}-x^{*}\right)\right) \\
& =\left(2 a^{\top} r+\lambda \theta \mathcal{N}\right) c_{2}^{\top}\left(x^{\prime}-x^{*}\right)+\lambda \theta \mathcal{N} c_{1}^{\top}\left(x^{\prime}-x^{*}\right)
\end{aligned}
$$

where we have used $a^{\top}\left(x^{\prime}-x^{*}\right)=0$ to obtain the first equality, $\mathcal{N}+2 c_{2}^{\top} r=0$ to obtain the third equality, and $\left(c_{1}-c_{2}\right)^{\top} r=\mathcal{N}$ to obtain the fifth equality. Now it follows from $2 a^{\top} r+\lambda \theta \mathcal{N}>0, c_{1}^{\top}\left(x^{\prime}-x^{*}\right)<0$, and $\lambda \theta \mathcal{N} \geq 0$ that $c_{2}^{\top}\left(x^{\prime}-x^{*}\right) \geq 0$.

Now suppose $\left(-a+\operatorname{cone}\left\{c_{1}, c_{2}\right\}\right) \cap \mathbb{L}^{n} \neq \emptyset$, and let $\lambda \geq 0$ and $0 \leq \theta \leq 1$ be such that $-a+\lambda\left(\theta c_{1}+(1-\theta) c_{2}\right) \in \mathbb{L}^{n}$. By Proposition 1, $x^{\prime}$ satisfies (6), and using (13), we can write

$$
\mathcal{N}\left(x^{\prime}-x^{*}\right)-2 c_{1}^{\top}\left(x^{\prime}-x^{*}\right) r \in \mathbb{L}^{n} .
$$

As before, because $\mathbb{L}^{n}$ is self-dual, we get

$$
0 \leq\left(-a+\lambda\left(\theta c_{1}+(1-\theta) c_{2}\right)\right)^{\top}\left(\mathcal{N}\left(x^{\prime}-x^{*}\right)-2 c_{1}^{\top}\left(x^{\prime}-x^{*}\right) r\right) .
$$

The right-hand side of this inequality is identical to

$$
\left(2 a^{\top} r+\lambda(1-\theta) \mathcal{N}\right) c_{1}^{\top}\left(x^{\prime}-x^{*}\right)+\lambda(1-\theta) \mathcal{N} c_{2}^{\top}\left(x^{\prime}-x^{*}\right)
$$

It follows from $2 a^{\top} r+\lambda(1-\theta) \mathcal{N}>0, c_{1}^{\top}\left(x^{\prime}-x^{*}\right)<0$, and $\lambda(1-\theta) \mathcal{N} \geq 0$ that $c_{2}^{\top}\left(x^{\prime}-x^{*}\right) \geq 0$. 
Finally suppose $\left(-a+\operatorname{cone}\left\{c_{2}\right\}\right) \cap-\mathbb{L}^{n} \neq \emptyset$, and let $\theta \geq 0$ be such that $-a+\theta c_{2} \in-\mathbb{L}^{n}$. Then using (15),

$$
\begin{aligned}
0 & \geq\left(-a+\theta c_{2}\right)^{\top}\left(\mathcal{N}\left(x^{\prime}-x^{*}\right)+2 c_{2}^{\top}\left(x^{\prime}-x^{*}\right) r\right) \\
& =-2 c_{2}^{\top}\left(x^{\prime}-x^{*}\right) a^{\top} r+\theta c_{2}^{\top}\left(x^{\prime}-x^{*}\right)\left(\mathcal{N}+2 c_{2}^{\top} r\right) \\
& =-2 c_{2}^{\top}\left(x^{\prime}-x^{*}\right) a^{\top} r .
\end{aligned}
$$

It follows from $a^{\top} r>0$ that $c_{2}^{\top}\left(x^{\prime}-x^{*}\right) \geq 0$.

b) If $a^{\top} r<0$, then $a^{\top}(-r)>0$. Since $-r=\left(\begin{array}{c}\tilde{c}_{2}-\tilde{c}_{1} \\ -c_{2, n}+c_{1, n}\end{array}\right)$, part (b) follows from part (a) by interchanging the roles of $C_{1}$ and $C_{2}$.

In the next result we show that the inequality (5) is sufficient to describe $\overline{\operatorname{conv}}\left(C_{1} \cup C_{2}\right)$ when conditions (11) and (12) hold.

Theorem 3. Let $C_{1}$ and $C_{2}$ be defined as in (2). Suppose Assumptions 3 and 4 hold. Let $c_{1}$ and $c_{2}$ be defined as in (10). Suppose also that one of the following conditions is satisfied:

a) $a^{\top} r=0$,

b) $a^{\top} r>0$ and 11 holds,

c) $a^{\top} r<0$ and $(12)$ holds.

Then

$$
\overline{\operatorname{conv}}\left(C_{1} \cup C_{2}\right)=\{x \in C: x \text { satisfies (5) }\} .
$$

Proof. Let $D$ denote the set on the right-hand side of (16). The inequality (5) is valid for $\overline{\operatorname{conv}}\left(C_{1} \cup C_{2}\right)$ by Theorem 1. Hence, $\overline{\operatorname{conv}}\left(C_{1} \cup C_{2}\right) \subseteq D$. Let $x^{\prime} \in D$. If $x^{\prime} \in C_{1} \cup C_{2}$, then clearly $x^{\prime} \in \overline{\operatorname{conv}}\left(C_{1} \cup C_{2}\right)$. Therefore, suppose $x^{\prime} \in C \backslash\left(C_{1} \cup C_{2}\right)$ is a point that satisfies (5). By Proposition 1, it satisfies (6) and (7) as well. We are going to show that in each case $x^{\prime}$ belongs to $\overline{\operatorname{conv}}\left(C_{1} \cup C_{2}\right)$.

a) Suppose $a^{\top} r=0$. By Remarks 2 and $3, \mathcal{N}=2 c_{1}^{\top} r=-2 c_{2}^{\top} r>0$. Define $\alpha_{1}, \alpha_{2}, x_{1}$, and $x_{2}$ as in (9). It is not difficult to see that $a^{\top} x_{1}=a^{\top} x_{2}=1$ and $c_{1}^{\top} x_{1}=c_{2}^{\top} x_{2}=0$. Furthermore, $x^{\prime} \in \operatorname{conv}\left\{x_{1}, x_{2}\right\}$ because $\alpha_{2}<0<\alpha_{1}$. One can show that $x_{1}, x_{2} \in \mathbb{L}^{n}$ using the same arguments as in the proof of Theorem 2. This proves $x_{1} \in C_{1}$ and $x_{2} \in C_{2}$.

b) Suppose $a^{\top} r>0$ and (11) holds. Let $x^{*}:=\frac{r}{a^{\top} r}$. Then by Lemma 2 , $c_{1}^{\top}\left(x^{\prime}-x^{*}\right)<0$ and $c_{2}^{\top}\left(x^{\prime}-x^{*}\right) \geq 0$.

First, suppose $c_{2}^{\top}\left(x^{\prime}-x^{*}\right)>0$, and let

$$
\begin{array}{rlrl}
\alpha_{1} & :=\frac{-c_{1}^{\top} x^{\prime}}{c_{1}^{\top}\left(x^{\prime}-x^{*}\right)}, & \alpha_{2} & :=\frac{-c_{2}^{\top} x^{\prime}}{c_{2}^{\top}\left(x^{\prime}-x^{*}\right)}, \\
x_{1} & :=x^{\prime}+\alpha_{1}\left(x^{\prime}-x^{*}\right), \quad x_{2}:=x^{\prime}+\alpha_{2}\left(x^{\prime}-x^{*}\right) .
\end{array}
$$


As in part a), $a^{\top} x_{1}=a^{\top} x_{2}=1, c_{1}^{\top} x_{1}=c_{2}^{\top} x_{2}=0$, and $x^{\prime} \in \operatorname{conv}\left\{x_{1}, x_{2}\right\}$ because $\alpha_{1}<0<$ $\alpha_{2}$. To show $x_{1}, x_{2} \in \mathbb{L}^{n}$, first note $\mathcal{N} x^{*}-2\left(c_{1}^{\top} x^{*}\right) r=\mathcal{N} x^{*}+2\left(c_{2}^{\top} x^{*}\right) r=0$ as in (13) and (14). Using this and $c_{1}^{\top} x_{1}=c_{2}^{\top} x_{2}=0$, we get

$$
\begin{aligned}
& \mathcal{N} x_{1}=\mathcal{N} x_{1}-2\left(c_{1}^{\top} x_{1}\right) r=\left(1+\alpha_{1}\right)\left(\mathcal{N} x^{\prime}-2\left(c_{1}^{\top} x^{\prime}\right) r\right), \\
& \mathcal{N} x_{2}=\mathcal{N} x_{2}+2\left(c_{2}^{\top} x_{2}\right) r=\left(1+\alpha_{2}\right)\left(\mathcal{N} x^{\prime}+2\left(c_{2}^{\top} x^{\prime}\right) r\right) .
\end{aligned}
$$

Clearly, $1+\alpha_{2}>0$, so $\mathcal{N} x_{2} \in \mathbb{L}^{n}$. Furthermore,

$$
1+\alpha_{1}=\frac{-c_{1}^{\top} x^{*}}{c_{1}^{\top}\left(x^{\prime}-x^{*}\right)}=\frac{-c_{1}^{\top} r}{\left(a^{\top} r\right) c_{1}^{\top}\left(x^{\prime}-x^{*}\right)}=\frac{-\mathcal{N}}{2\left(a^{\top} r\right) c_{1}^{\top}\left(x^{\prime}-x^{*}\right)}>0
$$

where we have used the relationships $\mathcal{N}>0, a^{\top} r>0$, and $c_{1}^{\top}\left(x^{\prime}-x^{*}\right)<0$ to reach the inequality. It follows that $\mathcal{N} x_{1} \in \mathbb{L}^{n}$ as well. Because $\mathcal{N}>0$, we get $x_{1}, x_{2} \in \mathbb{L}^{n}$. This proves $x_{1} \in C_{1}$ and $x_{2} \in C_{2}$.

Now suppose $c_{2}^{\top}\left(x^{\prime}-x^{*}\right)=0$, and define $\alpha_{1}$ and $x_{1}$ as in (17). All of the arguments that we have just used to show $\alpha_{1}<0$ and $x_{1} \in C_{1}$ continue to hold. Using $\mathcal{N} x^{*}+2 c_{2}^{\top} x^{*} r=0$, we can write

$$
\mathcal{N}\left(x^{\prime}-x^{*}\right)=\mathcal{N}\left(x^{\prime}-x^{*}\right)+2 c_{2}^{\top}\left(x^{\prime}-x^{*}\right) r \in \mathbb{L}^{n} .
$$

Because $\mathcal{N}>0$, we get $x^{\prime}-x^{*} \in \mathbb{L}^{n}$. Together with $c_{2}^{\top}\left(x^{\prime}-x^{*}\right)=0$ and $a^{\top}\left(x^{\prime}-x^{*}\right)=0$, this implies $x^{\prime}-x^{*} \in \operatorname{rec} C_{2}$. Then $x^{\prime}=x_{1}-\alpha_{1}\left(x^{\prime}-x^{*}\right) \in C_{1}+\operatorname{rec} C_{2}$ because $\alpha_{1}<0$. The claim now follows from the fact that the last set is contained in $\overline{\operatorname{conv}}\left(C_{1} \cup C_{2}\right)$ (see, e.g., [17, Theorem 9.8]).

c) Suppose $a^{\top} r<0$ and 12 holds. Since $-r:=\left(\begin{array}{c}\tilde{c}_{2}-\tilde{c}_{1} \\ -c_{2, n}+c_{1, n}\end{array}\right)$, part (c) follows from part (b) by interchanging the roles of $C_{1}$ and $C_{2}$.

The following result shows that when $C$ is an ellipsoid or a paraboloid, the closed convex hull of any two-term disjunction can be obtained by adding the cut (5) to the description of $C$.

Corollary 1. Let $C_{1}$ and $C_{2}$ be defined as in (2). Suppose Assumptions 3 and 4 hold. Let $c_{1}$ and $c_{2}$ be defined as in (10). If $a \in \mathbb{L}^{n}$, then (16) holds.

Proof. The result follows from Theorem 3 after observing that conditions (11) and (12) are trivially satisfied for any $c_{1}$ and $c_{2}$ when $a \in \mathbb{L}^{n}$.

The case of a split disjunction is particularly relevant in the solution of mixed-integer secondorder cone programs, and it has been studied by several groups recently, in particular Dadush et al. [10], Andersen and Jensen [1, Belotti et al. [4], and Modaresi et al. [16]. Theorem 3 has the following consequence for a split disjunction.

Corollary 2. Consider $C_{1}$ and $C_{2}$ defined by a split disjunction on $C$ as in (2). Suppose Assumptions 3 and 4 hold. Let $c_{1}$ and $c_{2}$ be defined as in (10). Then (16) holds. 
Proof. Let $l_{1}^{\top} x \geq l_{1,0} \vee l_{2}^{\top} x \geq l_{2,0}$ define a split disjunction on $C$ with $l_{2}=-t l_{1}$ for some $t>0$. Then we have $t l_{1,0}>-l_{2,0}$ so that $C_{1} \cup C_{2} \neq C$. Let $\lambda_{1}, \lambda_{2}, c_{1}$, and $c_{2}$ be defined as in (10). Let $\theta_{2}:=\frac{1}{\lambda_{2}\left(t l_{1,0}+l_{2,0}\right)}$ and $\theta_{1}:=\frac{t \lambda_{2} \theta_{2}}{\lambda_{1}}$. Then

$$
a+\theta_{1} c_{1}+\theta_{2} c_{2}=a+\lambda_{2} \theta_{2}\left(t\left(l_{1}-l_{1,0} a\right)+\left(l_{2}-l_{2,0} a\right)\right)=0 \in \mathbb{L}^{n} .
$$

The result now follows from Theorem 3 after observing that $\theta_{1}, \theta_{2} \geq 0$ implies that conditions (11) and (12) are satisfied.

When the sets $C_{1}$ and $C_{2}$ do not intersect, except possibly on their boundary, Proposition 1 says that (5) can be expressed in conic quadratic form and directly implies the following result.

Corollary 3. Let $C_{1}$ and $C_{2}$ be defined as in (2). Suppose Assumptions 3 and 4 hold. Let $c_{1}$ and $c_{2}$ be defined as in (10). Suppose that one of the conditions a), b), or c) of Theorem 3 holds. Suppose, in addition, that

$$
\left\{x \in C: c_{1}^{\top} x>0, c_{2}^{\top} x>0\right\}=\emptyset .
$$

Then

$$
\begin{aligned}
\overline{\operatorname{conv}}\left(C_{1} \cup C_{2}\right) & =\{x \in C: x \text { satisfies }(6)\} \\
& =\{x \in C: x \text { satisfies }(7)\} .
\end{aligned}
$$

Remark 4. Conditions (11) and (12) are directly related to the sufficient conditions that guarantee the closedness of the convex hull of a two-term disjunction on $\mathbb{L}^{n}$ explored in [14]. In particular, one can show that the convex hull of a disjunction $h_{1}^{\top} x \geq h_{1,0} \vee h_{2}^{\top} x \geq h_{2,0}$ on the whole second-order cone $\mathbb{L}^{n}$ is closed if

i) $h_{1,0}=h_{2,0} \in\{ \pm 1\}$ and there exists $0<\mu<1$ such that $\mu h_{1}+(1-\mu) h_{2} \in \mathbb{L}^{n}$, or

ii) $h_{1,0}=h_{2,0}=-1$ and $h_{1}, h_{2} \in-\operatorname{int} \mathbb{L}^{n}$.

In our present context, exploiting $(i)$ and $(i i)$ after letting $h_{i}:=a+\theta_{i} c_{i}$ and $h_{i, 0}:=1$ (or, $h_{i}:=-a+\theta_{i} c_{i}$ and $\left.h_{i, 0}:=-1\right)$ for some $\theta_{i}>0$ leads to (11) and (12).

\subsection{Two Examples}

In this section we illustrate Theorem 3 with two examples.

\subsubsection{A Two-Term Disjunction on a Paraboloid}

Consider the disjunction $-2 x_{1}-x_{2}-2 x_{4} \geq 0 \vee x_{1} \geq 0$ on the paraboloid $C:=\left\{x \in \mathbb{L}^{4}\right.$ : $\left.x_{1}+x_{4}=1\right\}$. Let $C_{1}:=\left\{x \in C:-2 x_{1}-x_{2}-2 x_{4} \geq 0\right\}$ and $C_{2}:=\left\{x \in C: x_{1} \geq 0\right\}$. Noting that $C$ is a paraboloid and $C_{1}$ and $C_{2}$ are disjoint, we can use Corollary 3 to characterize $\overline{\operatorname{conv}}\left(C_{1} \cup C_{2}\right)$ with a conic quadratic inequality:

$$
\overline{\operatorname{conv}}\left(C_{1} \cup C_{2}\right)=\left\{x \in C: 3 x+x_{1}(-3 ;-1 ; 0 ; 2) \in \mathbb{L}^{4}\right\} .
$$

Figure 1 depicts the paraboloid $C$ in mesh and the disjunction $C_{1} \cup C_{2}$ in blue. The conic quadratic disjunctive cut added to convexify this set is shown in red. 


\subsubsection{A Two-Term Disjunction on a Hyperboloid}

Consider the disjunction $-2 x_{1}-x_{2} \geq 0 \vee \sqrt{2} x_{1}-x_{3} \geq 0$ on the hyperboloid $C:=\left\{x \in \mathbb{L}^{3}\right.$ : $\left.x_{1}=2\right\}$. Let $C_{1}:=\left\{x \in C:-2 x_{1}-x_{2} \geq 0\right\}$ and $C_{2}:=\left\{x \in C: \sqrt{2} x_{1}-x_{3} \geq 0\right\}$. Note that, in this setting,

$$
a^{\top} r=\frac{1}{10}(1 ; 0 ; 0)^{\top}(-2 \sqrt{5}+5 \sqrt{2} ;-\sqrt{5} ;-5)<0,
$$

but none of the conditions (12) are satisfied. The conic quadratic inequality

$$
(5+2 \sqrt{10}) x+\left(\sqrt{2} x_{1}-x_{3}\right)(-2 \sqrt{5}+5 \sqrt{2} ;-\sqrt{5} ;-5) \in \mathbb{L}^{3}
$$

of Theorem 3 is valid for $C_{1} \cup C_{2}$ but not sufficient to describe its closed convex hull. Indeed, the inequality $x_{2} \leq 2$ is valid for $\overline{\operatorname{conv}}\left(C_{1} \cup C_{2}\right)$ but is not implied by (18). Figure 2 depicts the hyperboloid $C$ in mesh and the disjunction $C_{1} \cup C_{2}$ in blue. The conic quadratic disjunctive cut (18) is shown in red.

\section{Acknowledgments}

We would like to thank Fatma Kılınç-Karzan for the fruitful discussions that led to the results presented in this paper. This work was supported in part by NSF grant CMMI1263239 and ONR grant N00014-12-10032.

\section{References}

[1] K. Andersen and A. N. Jensen. Intersection cuts for mixed integer conic quadratic sets. In Proceedings of IPCO 2013, volume 7801 of Lecture Notes in Computer Science, pages 37-48, Valparaiso, Chile, March 2013.

[2] A. Atamtürk and V. Narayanan. Conic mixed-integer rounding cuts. Mathematical Programming, 122:1-20, 2010. 
[3] E. Balas. Intersection cuts - a new type of cutting planes for integer programming. Operations Research, 19:19-39, 1971.

[4] P. Belotti, J. C. Goez, I. Polik, T. K. Ralphs, and T. Terlaky. A conic representation of the convex hull of disjunctive sets and conic cuts for integer second order cone optimization. Technical report, Department of Industrial and Systems Engineering, Lehigh University, Bethlehem, PA, June 2012.

[5] P. Belotti, J.C. Góez, I. Pólik, T.K. Ralphs, and T. Terlaky. On families of quadratic surfaces having fixed intersections with two hyperplanes. Discrete Applied Mathematics, 161:2778-2793, 2013.

[6] D. Bienstock and A. Michalka. Cutting-planes for optimization of convex functions over nonconvex sets. SIAM Journal on Optimization, 24:643-677, 2014.

[7] P. Bonami. Lift-and-project cuts for mixed integer convex programs. In Proceedings of IPCO 2011, volume 6655 of Lecture Notes in Computer Science, pages 52-64, New York, NY, June 2011.

[8] S. Burer and F. Kılınç-Karzan. How to convexify the intersection of a second-order cone with a non-convex quadratic. Working paper, June 2014.

[9] M. Çezik and G. Iyengar. Cuts for mixed 0-1 conic programming. Mathematical Programming, 104:179-202, 2005.

[10] D. Dadush, S. S. Dey, and J. P. Vielma. The split closure of a strictly convex body. Operations Research Letters, 39:121-126, 2011.

[11] S. Drewes. Mixed Integer Second Order Cone Programming. PhD thesis, Technische Universität Darmstadt, 2009.

[12] S. Drewes and S. Pokutta. Cutting-planes for weakly-coupled 0/1 second order cone programs. Electronic Notes in Discrete Mathematics, 36:735-742, 2010. 
[13] F. Kılınç-Karzan. On minimal valid inequalities for mixed integer conic programs. Mathematics of Operations Research, 2015, to appear.

[14] F. Kılınç-Karzan and S. Yıldız. Two-term disjunctions on the second-order cone. Mathematical Programming B, 2015, to appear.

[15] S. Modaresi, M. R. Kılınç, and J. P. Vielma. Split cuts and extended formulations for mixed integer conic quadratic programming. Operations Research Letters, 43:10-15, 2015.

[16] S. Modaresi, M. R. Kılınç, and J. P. Vielma. Intersection cuts for nonlinear integer programming: Convexification techniques for structured sets. Mathematical Programming, 2015, to appear.

[17] R. T. Rockafellar. Convex Analysis. Princeton Landmarks in Mathematics. Princeton University Press, New Jersey, 1970.

[18] R. A. Stubbs and S. Mehrotra. A branch-and-cut method for 0-1 mixed convex programming. Mathematical Programming, 86:515-532, 1999. 